on his analysis of the studies of Gaze and his co-workers on the formation of retino-tectal connexions in amphibians. This work has shown that connexions are formed along two developmental axes-dorso-ventral and naso-temporaldetermined at different stages in the growth of the tadpole. Goodwin pointed out that the diffusion model with "morphogen" gradients proposed by Gaze et al. (J. Physiol., 165, 484; 1963) does not account for all the experimental data from their work with compound eyes. In particular, there is a mysterious kink which appears in one of the axes when a "double ventral" eye is created by grafting the ventral half of one retina to that of the other at a stage of development between the determination of the two axes.

Goodwin went on to show how this distortion could be explained in terms of a model of development based on frequency gradients in periodic signals propagating across the embryonic field (B. C. Goodwin and M. H. Cohen, J. Theoret. Biol., 25, 49; 1969). Assuming signal sources at $90^{\circ}$ to each other, the model predicts an interaction between the two axes which would produce the observed kink. Goodwin pointed out, however, that any model with two interacting axes $90^{\circ}$ apart would equally explain this finding. The specific model he used could only be vindicated by further research showing periodic movements of the developing cells.

No such diffidence characterized the presentation of $\mathrm{Mr}$ A. D. J. Robertson (University of Chicago), who has interpreted, from the mass of literature and films, the aggregation of the cellular slime mould Dictyostelium discoideum. Although the life cycle of this organism, which entails the formation of a slug from initially independent amoebae, provides a beautifully simple developmental paradigm, it has so far eluded explanation. Robertson's dogma was that not only could a model with frequency gradients in periodic signals elegantly explain many of the bewildering observations on slime mould aggregation, but periodicity and pulsatile movement of the amoebae could actually be seen on film. After describing the aggregation of $D$. discoideum in the conceptual framework of a mathematical model developed in collaboration with Professor M. H. Cohen (J. Theoret. Biol., in the press), he went on to illustrate his points with just such a film; and concluded with the reassurance that if the phenomena he wished to emphasize were not immediately convincing, this was because real conviction could only be acquired after repeated observation with a closed mind.

The contribution which followed underlined the unfortunate truth that it is not difficult to make models: the difficulty lies in finding the right one. Dr L. A. Segel (Troy Polytechnic Insti- tute) approached the problem of slime mould aggregation as an expert on heat equations. Points of agreement with the Robertson-Cohen doctrine were apparent : aggregation is guided by chemotaxis, for which the attractant (acrasin) is cyclic adenosine monophosphate (cyclic AMP), secreted by the amoebae along with an enzyme (acrasinase) which rapidly destroys it. But whereas the model of Cohen and Robertson depends on the assumption of essentially pulsatile production of cyclic AMP, that of Segel assumes that production is continuous. His amoebae aggregate where random movement has resulted in chance clusters in which the concentration of cyclic AMP is too great to be efficiently destroyed by the acrasinase.

Dr R. B. Stein (University of Alberta), speaking on the properties of neuronal spike trains, based his conclusions on the behaviour of a concrete or, more precisely, an electronic model. He used the response of ả model neurone to sinusoidal stimulation, with and without random background noise, to illustrate a possible adaptive function for random variation in the nervous system. With low frequency stimulation and no background noise, the inpulse response tended to be phase locked (as in the real auditory system); but with increasing frequency, both phase and frequency information were lost because the firing frequency of the neurone is limited by the time course of membrane capacitance discharge. With added noise, however, the membrane is maintained close to threshold; and when the response is averaged over time, the probability of firing of the neurone emerges as a fairly precise function of the input. Thus by averaging over neurones instead of over time, a reliable response can be seen tó be produced from a noisy system; and further support is provided for an idea that is far from new to brain research.

The usual justifications for model making in biology are that it is necessary for a proper understanding of complex systems, and that it makes possible quantitative predictions which can be rigorously tested. Dr B. Julesz (Bell Telephone Laboratories) must be the only living theoretician who can also claim to have created a spectacle whose visual impact unfailingly causes nonspecialist audiences to gasp aloud. His talk on his experiments in binocular depth perception with computer-generated random patterns was liberally illustrated with slides and films from which proof of his theory literally leapt out. There was no need for Julesz to labour his point : seeing is believing.

\section{Fusing Cells in Mice}

INNUMERABLE experiments have now been performed to fuse together diverse types of cell in vitro by using inactivated Sendai virus. The hybrid cells produced by fusion of two normal cells possess a joint cytoplasm, but retain, for a while, the individual nuclei of the parent cells (although these may subsequently fuse to form one nucleus). One of the most interesting aspects of hybrid cell formation is that a cell with a defect in, for example, some enzyme activity can be "cured" by fusion with another cell type which contains the missing activity. A new and rather complicated way to test for this "gene complementation" is reported by N. L. Levy and R. L. Ladda in next Wednesday's issue of Nature New Biology (229, 1971).

"Old" mice and "new" mice differ at only one genetic locus, which specifies a protein which confers haemolytic complement activity on the animal. "Old" mice can have haemolytic complement activity restored by the injection of bone marrow cells from the "new" strain and this activity probably results from migration of the injected cells to the spleen, where they elaborate the missing protein. Levy and Ladda have used the restoration of haemolytic complement activity in a "new" mouse injected with hybrid cells as a test to see whether the hybrid cells can make the missing protein activity.

Hybrid cells were made by fusing together different types of mouse or chick cells by the usual treatment with Sendai virus. The injection of "new" mouse kidney : "old" mouse spleen or "old" mouse spleen : chick erythrocyte hybrids restored haemolytic complement activity to deficient mice. This result means that the hybrid cells must be able to synthesize the gene product in which the new mice are defective. This is not too surprising in the former case, because one of the parent cell types derives from "new" mice which have the activity. The other result cannot be explained in this way, however. Because little chick cytoplasm is incorporated into chick erythrocyte : mammalian cell hybrids, it seems likely that it is the presence of the chick nucleus in the second set which enables the hybrids to make the missing complement activity.

There seem to be two ways in which this could happen. Levy and Ladda do not know whether the missing activity was filled by a chick protein (which would be specified by the chick nucleus but be able to perform the same catalytic functions as the mouse-specified protein), or whether the hybrid can make the mouse protein itself. The former could result from activation of the chick nucleus in the hybrid cell. The alternative explanation would imply that the mutation which causes the "old" mouse phenotype affects a control gene and not the structural gene which codes for the missing enzyme. 